ACMG STATEMENT

\title{
Direct-to-consumer prenatal testing for multigenic or polygenic disorders: a position statement of the American College of Medical Genetics and Genomics (ACMG)
}

\author{
ACMG Board of Directors ${ }^{1 *}$ \\ Genetics in Medicine (2021) 23:2027-2028; https://doi.org/10.1038/s41436-021-01247-1
}

\begin{abstract}
Disclaimer: This statement is designed primarily as an educational resource for medical geneticists and other clinicians to help them provide quality medical services. Adherence to this statement is completely voluntary and does not necessarily assure a successful medical outcome. This statement should not be considered inclusive of all proper procedures and tests or exclusive of other procedures and tests that are reasonably directed to obtaining the same results. In determining the propriety of any specific procedure or test, the clinician should apply his or her own professional judgment to the specific clinical circumstances presented by the individual patient or specimen.

Clinicians are encouraged to document the reasons for the use of a particular procedure or test, whether or not it is in conformance with this statement. Clinicians also are advised to take notice of the date this statement was adopted, and to consider other medical and scientific information that becomes available after that date. It also would be prudent to consider whether intellectual property interests may restrict the performance of certain tests and other procedures.
\end{abstract}

The American College of Medical Genetics and Genomics' (ACMG's) updated position statement on direct-to-consumer testing (2016) [1] noted, in part, that:

- A knowledgeable professional should be involved in the process of ordering a genetic test with medical implications.

- Laboratory results should be interpreted and delivered by a board-certified genetics professional.

- A genetics professional should be available to help the consumer determine, for example, whether a genetic test should be performed and how to interpret test results in light of personal and family history.

- The scientific evidence base describing the validity and utility of a genetic test should be clearly stated.

- The consumer should be fully informed regarding what the test can and cannot say about his or her health, since many directto-consumer genetic tests do not give a definitive answer regarding whether an individual will develop a given condition.

While the issues surrounding direct-to-consumer testing for monogenic disease or disease caused by copy-number variants are complicated, they are even more complex for disorders that exhibit multigenic or polygenic inheritance. These disorders, by definition, have been shown to be controlled in part by multiple genetic loci, sometimes with an influence of environmental factors that are often unknown. Examples include (but are not limited to) psychiatric disease (especially schizophrenia), cardiovascular disease, complex heart disease, diabetes mellitus (especially type 2), and Alzheimer disease.

Techniques to analyze the genetic components contributing to multi/polygenic disease have evolved considerably over the past several decades, having first been defined largely by pedigree analysis and family studies, then genome-wide association studies, and most recently by exome and genome sequencing. However, even with the most sophisticated technologies available, genetic studies on complex traits and disease constitute an inexact science and do not identify affected individuals as does testing for monogenic disorders. Rather, it only assesses the risk for developing a disorder.

As a result of increasing data points, the need for interpretation of and counseling about any type of genetic testing regarding these disorders has become exponentially more complex, and in most instances, is not a part of general clinical genetics practice. In this context, the emergence of direct-to-consumer testing for multi/polygenic diseases is particularly troubling. This is especially so when applied as a prenatal test to mitigate genetic risk through selective embryo termination, since a definitive and specific genetic diagnosis cannot be made, only risk inferred. As such, the ACMG is of the opinion that prenatal testing for disorders that exhibit multigenic or polygenic inheritance is not yet appropriate for clinical use and should not be offered as direct-to-consumer testing.

Received: 1 June 2021; Revised: 1 June 2021; Accepted: 3 June 2021

Published online: 28 June 2021

\section{REFERENCE}

1. ACMG Board of Directors. Direct-to-consumer genetic testing: a revised position statement of the American College of Medical Genetics and Genomics. Genet. Med. 2016;18:207-8.

\section{COMPETING INTERESTS}

The authors declare no competing interests.

\footnotetext{
'American College of Medical Genetics and Genomics, Bethesda, MD, USA. *The Board of Directors of the American College of Medical Genetics and Genomics approved this

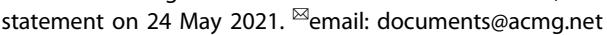




\section{ADDITIONAL INFORMATION}

Correspondence and requests for materials should be addressed to ACMG.

Reprints and permission information is available at http://www.nature.com/ reprints
Publisher's note Springer Nature remains neutral with regard to jurisdictional claims in published maps and institutional affiliations. 